\title{
Ultracompact Monostatic MIMO Radar With Nonredundant Aperture
}

\author{
Patrik Grüner ${ }^{\circledR}$, Graduate Student Member, IEEE, Martin Geiger ${ }^{\circledR}$, Graduate Student Member, IEEE, \\ and Christian Waldschmidt ${ }^{(}$, Senior Member, IEEE
}

\begin{abstract}
Monostatic multiple-input-multiple-output (MIMO) radar configurations using the same antenna array for transmission and reception inherently lead to redundant virtual antenna positions within the virtual MIMO aperture. This article presents a new method for designing monostatic MIMO radars where this disadvantage may be overcome and nonredundant virtual arrays are yielded by exploiting biomimetic antenna arrays (BMAAs). The new concept of biomimetic MIMO is introduced and thoroughly investigated in this article. Its working principle is compared with conventional monostatic MIMO and verified by radar measurements. The new approach is intended to improve the angle estimation capability of, in particular, small radar systems. Therefore, the concept is applied to an ultracompact twochannel radar monolithic microwave integrated circuit (MMIC) with fully integrated antennas for range and angle measurements in the $150-\mathrm{GHz}$ range. The chip features two monostatic transmit-receive (TRX) channels with an antenna spacing of $\lambda / 4$ and occupies less than $3 \mathrm{~mm}^{2}$ of space. By applying biomimetic MIMO, a virtual aperture consisting of four unique antenna positions and an equivalent total size of around $1.5 \lambda$ is achieved.
\end{abstract}

Index Terms-Biomimetic antenna array (BMAA), biomimetic multiple-input-multiple-output (MIMO), direction-ofarrival (DOA) estimation, frequency-modulated continuous-wave (FMCW), MIMO radar, radar monolithic microwave integrated circuit (MMIC), radar-on-chip.

\section{INTRODUCTION}

$\mathbf{T}$ $\mathrm{HE}$ advancement in semiconductor technology enables the integration of radar sensors on a single chip [1], [2]. The demand for high range resolution and therefore high absolute bandwidth drives the development of radars to higher carrier frequencies. In the frequency range above $100 \mathrm{GHz}$, the antennas become comparatively small, and it is possible to integrate them directly on the monolithic microwave integrated circuit (MMIC). As a consequence, no RF signals have to be routed from or to the MMIC through lossy bond connections. However, if more and more antennas are to be integrated directly on the MMIC to increase the angular resolution of the radar, methods are necessary to limit the required chip area in order to save costs.

Manuscript received February 5, 2020; revised April 7, 2020; accepted May 29, 2020. Date of publication July 13, 2020; date of current version November 4, 2020. This work was supported by the German Research Foundation [Deutsche Forschungsgemeinschaft (DFG)] under Grant WA 3506/6-1. (Corresponding author: Patrik Grüner.)

The authors are with the Institute of Microwave Engineering, Ulm University, 89081 Ulm, Germany (e-mail: pgruener@ieee.org).

Color versions of one or more of the figures in this article are available online at http://ieeexplore.ieee.org.

Digital Object Identifier 10.1109/TMTT.2020.3006055
Several designs of single-channel frequency-modulated continuous-wave (FMCW) radar sensors above $100 \mathrm{GHz}$ with antennas on chip or in package were presented in the past years [3]-[8]. However, when integrating a complete radar-onchip, the antennas are typically the largest single components on the MMIC. If an ultracompact design is desired in order to save expensive chip area, a monostatic design with only one common antenna for transmitting (TX) and receiving (RX) is therefore preferred [9]-[11].

When it comes to measuring not only distance and velocity but also the angle of a target relative to the radar, at least two RX channels are required to evaluate the phase progression of the incident plane wave between the antennas. The distance between the respective antennas is of great importance since a large spacing results in a high angular estimation performance. However, when implementing on an MMIC, the large antenna size as well as the large antenna spacing lead to a significant increase in the chip area, which makes the MMIC more and more expensive. The placement of the antennas off the MMIC on the printed circuit board (PCB), such as it is done in [12] and [13], should be avoided for frequencies well above $100 \mathrm{GHz}$ since the bond connections introduce high losses and parasitic radiation. If, on the other hand, the antennas are integrated directly on the MMIC, this results in a large chip area [14], [15].

One possible approach to reduce the required chip area is the recently introduced concept of biomimetic antenna arrays (BMAAs). Two antenna elements are coupled in such a way that they mimic the hearing system of the fly Ormia ochracea [16]-[19]. The result is a significantly increased angle estimation performance of the antenna array at the expense of a reduction in the signal-to-noise ratio. The BMAA antenna system allows either the enhancement of the angle estimation of an existing antenna array [20] or the spacing between the single antenna elements to be smaller while maintaining the angular performance of the unmodified but larger array.

A method to further increase the angle estimation performance especially in terms of angular separability is to use the radar in a multiple-input-multiple-output (MIMO) configuration [21]. A spatial convolution of transmit and receive apertures leads to a virtual aperture being larger than the sum of the TX and RX apertures. However, when combining an MIMO radar system for high angular resolution with the compactness of a monostatic radar system, the resulting virtual 


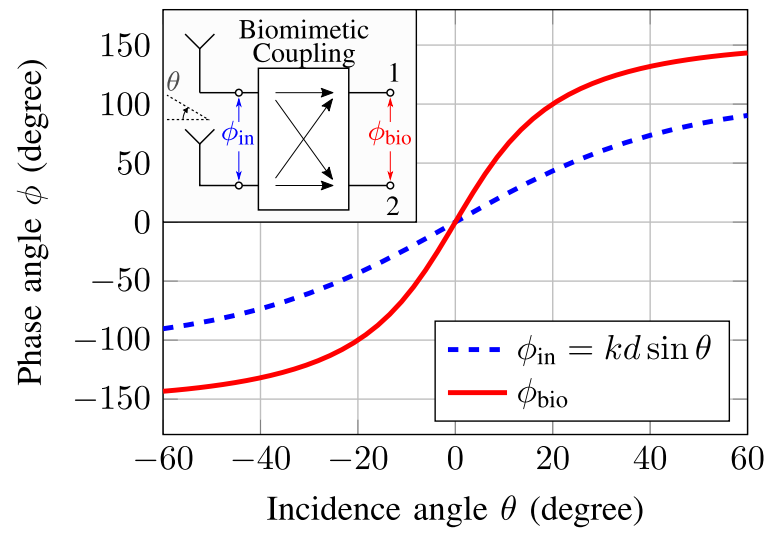

(a)

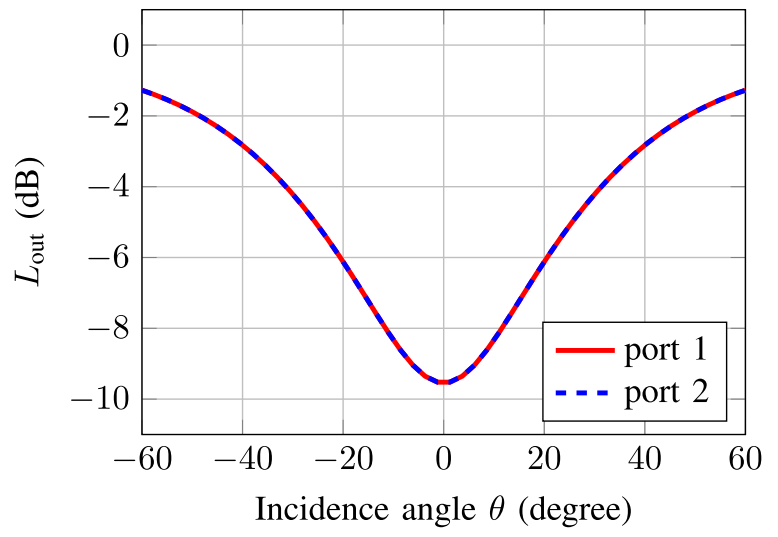

(b)

Fig. 1. (a) Theoretical phase difference curves and (b) output power level $L_{\text {out }}$ for a BMAA with antenna separation $d=0.37 \lambda$, phase gain $\eta=3$, and $L_{\text {out }}=-9.5 \mathrm{~dB}$. The two curves for $L_{\text {out }}$ are identical and lie on top of each other [18].

MIMO aperture inherently contains redundant virtual antenna positions, which do not deliver additional information. The same holds for bistatic radars if TX and RX antennas are spaced identically [15].

This article presents an ultracompact two-channel monostatic radar MMIC that enables angular measurements on an extremely small footprint. A fundamentally new MIMO operation is demonstrated by placing the biomimetic coupling of the on-chip BMAA only in the receive path of the monostatic radar, which avoids the redundancy of conventional monostatic MIMO arrays, increases the unambiguous angular range, and improves the angular resolution. For the first time, the concept of the BMAA is applied on an MMIC and above $100 \mathrm{GHz}$ in order to reduce the necessary chip area and improve the angle estimation capability. This article is organized as follows. Section II presents a short summary of the BMAA and introduces the concept of biomimetic MIMO. Section III describes the system design of the ultracompact two-channel radar MMIC in the $150-\mathrm{GHz}$ range applying the biomimetic MIMO concept. Radar measurements were conducted, and the results are presented in Section IV.

\section{Concept of Nonredundant Monostatic Mimo}

\section{A. Biomimetic Antenna Array}

In this section, a brief overview of the BMAA as a concept to increase the angle estimation performance for electrically small apertures is given. For the sake of compactness, only the effects on a system level are considered and parameters are defined to quantify them. For a detailed explanation of the underlying working principle, please refer to [18].

A wave incident from an angle $\theta$ leads to a phase progression

$$
\phi_{\text {in }}=k d \sin \theta:=2 \alpha
$$

at the antenna terminals of two antennas arranged at a distance $d$ according to the inset in Fig. 1(a), where $k=2 \pi / \lambda$ is the wavenumber in free space and $\lambda$ is the wavelength. Due to the biomimetic coupling, the phase difference $\phi_{\text {bio }}$ after the biomimetic coupling (BMC) network is significantly enlarged compared with $\phi_{\text {in }}$ [18]. An exemplary course of the phase progression is shown in Fig. 1(a). The increased phase sensitivity comes at the cost of a reduction in RX power, which can be extracted from the array [see Fig. 1(b)].

The biomimetic antenna system consisting of two antennas and one coupling network can be fully described on system level by only three parameters: the phase gain $\eta$, the offboresight factor $\xi$, and the normalized output power $L_{\text {out }}$ [18], [22], [23]. The phase gain and the normalized output power compare the BMAA to a regular antenna with the same antenna elements at the same spacing but without the coupling network. The phase gain is defined in the boresight direction by normalizing the slope of the phase progression at the terminal of the BMAA $\left(\phi_{\text {bio }}\right)$ to the slope of the regular antenna array phase progression $\left(\phi_{\text {in }}\right)$

$$
\eta=\frac{\left.\frac{\mathrm{d} \phi_{\mathrm{bio}}(\theta)}{\mathrm{d} \theta}\right|_{\theta=0}}{\left.\frac{\mathrm{d} \phi_{\mathrm{in}}(\theta)}{\mathrm{d} \theta}\right|_{\theta=0}} .
$$

The phase gain basically describes the increase in steepness of the BMAA phase difference compared with the regular antenna array phase difference steepness. The reduction of the output power is quantified by the dimensionless quantity $L_{\text {out }}$

$$
L_{\text {out }}=\frac{P_{\text {out,BMAA }}}{P_{\text {out,conv. Array }}} .
$$

This value is always less than or equal to 1 . The off-boresight factor $\xi$ quantifies the shift in the position of maximum steepness of the phase progression to incidence angles other than boresight (i.e., $|\theta|>0$ ) [23]. However, without loss of generality in this article, only boresight BMAAs are assumed, and therefore, $\xi$ is always considered as 0 .

Based on the introduced BMAA parameters, an analytical expression for the phase difference $\phi_{\text {bio }}$ after the biomimetic coupling can be derived as [17]

$$
\phi_{\text {bio }}=\arctan \left(\frac{2 \eta \tan \alpha}{1-\left(\eta^{2}+\xi^{2}\right) \tan ^{2} \alpha}\right)
$$

with the phase gain $\eta$ in the boresight direction and the offboresight factor $\xi$. The corresponding normalized power level 


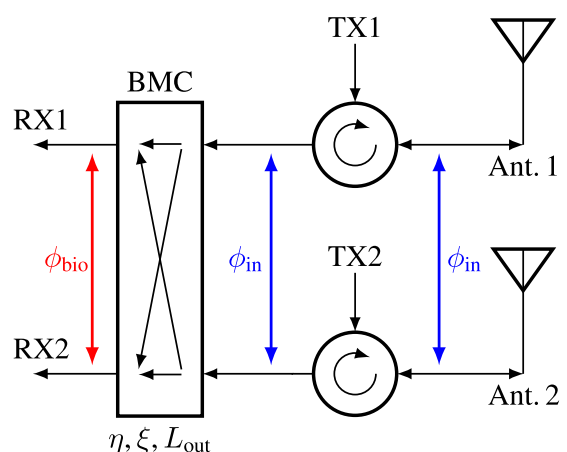

Fig. 2. Monostatic radar system with biomimetic coupling in the RX path only. $\phi_{\text {in }}$ is the phase difference due to the physical spacing of the antennas, whereas $\phi_{\text {bio }}$ is the phase difference after the biomimetic coupling.

calculates to (see Appendix)

$$
L_{\text {out }}=\frac{|1 \pm \mathrm{j}(\eta+\mathrm{j} \xi) \tan \alpha|^{2}}{|(\eta+\mathrm{j} \xi)+\mathrm{j}(\eta+\mathrm{j} \xi) \tan \alpha|^{2}} .
$$

The course of the phase progression and the output power level in Fig. 1 corresponds to a BMAA with an antenna spacing of $d=0.37 \lambda$, a phase gain of $\eta=3$, and a minimum normalized power level of $L_{\text {out }}=-9.5 \mathrm{~dB}$.

\section{B. Biomimetic MIMO Antenna System}

A well-known method to increase the aperture size is using a radar with multiple transmitters and receivers in MIMO mode [15], [21]. The transmitted signals have to be orthogonal so that they can be distinguished from each other at the receivers. This orthogonality can be established by means of time, frequency, or code multiplexing. If the orthogonality criterion is fulfilled, the antenna apertures of the transmitter and the receiver form a virtual aperture, which is calculated by a spatial convolution of the two apertures [24].

When considering a monostatic radar system with two antennas as it is shown in Fig. 2, the size of the TX aperture is inevitably identical to the size of the RX aperture as the very same antennas are used. As a consequence, the array steering vectors in both cases are identical and can be modeled by

$$
\boldsymbol{a}_{\mathrm{RX}}(\theta)=\boldsymbol{a}_{\mathrm{TX}}(\theta)=\left(1, \mathrm{e}^{-\mathrm{j} \phi_{\mathrm{in}}}\right)^{\mathrm{T}} .
$$

Each element of the steering vector is normalized to the first array element. The resulting steering vector of the virtual array in the monostatic MIMO case is given by

$$
\boldsymbol{a}(\theta)=\left(1, \mathrm{e}^{-\mathrm{j} \phi_{\text {in }}}, \mathrm{e}^{-\mathrm{j} \phi_{\text {in }}}, \mathrm{e}^{-\mathrm{j} 2 \phi_{\text {in }}}\right)^{\mathrm{T}}
$$

where $\boldsymbol{a}(\theta)=\boldsymbol{a}_{\mathrm{TX}}(\theta) \otimes \boldsymbol{a}_{\mathrm{RX}}(\theta)$ is the Kronecker product of the steering vectors of the transmitter and the receiver aperture [24]. While the steering vector consists of four entries, the actual virtual aperture consists of only three elements because one element is redundant and does not deliver additional information. The transmitter and receiver apertures as well as the resulting virtual aperture with the redundant element are shown in Fig. 3(a).

By comparing the phase progressions of biomimetic and conventional arrays in Fig. 1, the steeper phase progression in the biomimetic case may be regarded as a larger antenna

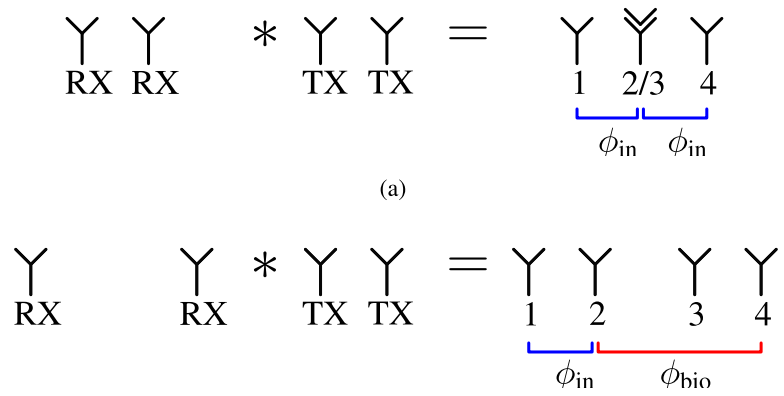

(b)

Fig. 3. Formation of the virtual MIMO aperture in the boresight direction for (a) conventional monostatic antenna array and (b) biomimetic monostatic antenna array with biomimetic coupling in the RX path only.

element spacing. This enables a fundamentally new architecture for monostatic MIMO radar systems. By using the BMC only in the RX path of the radar, the RX aperture seems to be larger than the TX aperture. Mathematically, the steering vectors in the TX and RX case are

$$
\begin{aligned}
\boldsymbol{a}_{\mathrm{TX}}(\theta) & =\left(1, \mathrm{e}^{-\mathrm{j} \phi_{\text {in }}}\right)^{\mathrm{T}} \\
\boldsymbol{a}_{\mathrm{RX}, \text { bio }}(\theta) & =\left(1, \mathrm{e}^{-\mathrm{j} \phi_{\text {bio }}}\right)^{\mathrm{T}} .
\end{aligned}
$$

The resulting virtual array steering vector for the MIMO operation, normalized to the first array element, is then given by

$$
\boldsymbol{a}_{\text {bio }}(\theta)=\left(1, \mathrm{e}^{-\mathrm{j} \phi_{\text {bio }}}, \mathrm{e}^{-\mathrm{j} \phi_{\text {in }}}, \mathrm{e}^{-\mathrm{j}\left(\phi_{\text {in }}+\phi_{\text {bio }}\right)}\right)^{\mathrm{T}}
$$

where $a_{\mathrm{bio}}(\theta)=a_{\mathrm{TX}}(\theta) \otimes a_{\mathrm{RX}, \mathrm{bio}}(\theta)$. The resulting virtual aperture no longer contains redundant elements but four individually placed virtual antenna elements. The BMAA principle allows the scaling of the RX aperture, whereas the physical separation of the two antenna elements remains constant. As a consequence, the virtual array is built up of four virtual antenna elements, whereas only two physical antenna elements are necessary. The principle and the resulting virtual aperture are shown in Fig. 3(b).

The antenna system performance is evaluated by the ambiguity function (AF) defined as follows [25]:

$$
\operatorname{AF}\left(\theta_{i}, \theta_{j}\right)=\frac{\boldsymbol{a}\left(\theta_{i}\right)^{\mathrm{H}} \cdot \boldsymbol{a}\left(\theta_{j}\right)}{\left\|\boldsymbol{a}\left(\theta_{i}\right)\right\|\left\|\boldsymbol{a}\left(\theta_{j}\right)\right\|}
$$

where $(\cdot)^{\mathrm{H}}$ denotes the Hermitian operator and $\|\cdot\|$ denotes the Euclidean norm of a vector. The AF is a measure of how similar the steering vectors are for two incidence angles $\theta_{i}$ and $\theta_{j}$ and takes values between 0 and 1. Fig. 4(a) shows the AF of the conventional antenna array according to the virtual array steering vector in (7), whereas Fig. 4(b) shows the same for the system with biomimetic coupling with a phase gain of $\eta=3$ in the RX path according to (10). A physical antenna spacing of $d=0.37 \lambda$ was assumed in both cases. A significantly narrower main lobe around the boresight direction $\left(\theta_{i}=0^{\circ}\right)$ due to the enlarged aperture can be recognized $\left(\theta_{3 \mathrm{~dB}, \mathrm{BMAA}}=29^{\circ}\right.$ versus $\theta_{3 \mathrm{~dB} \text {, conv }}=58^{\circ}$ ). The peak phase gain is established in the boresight direction but decays with increasing incidence angle $\theta$. As a consequence, no ambiguity occurs. For very 


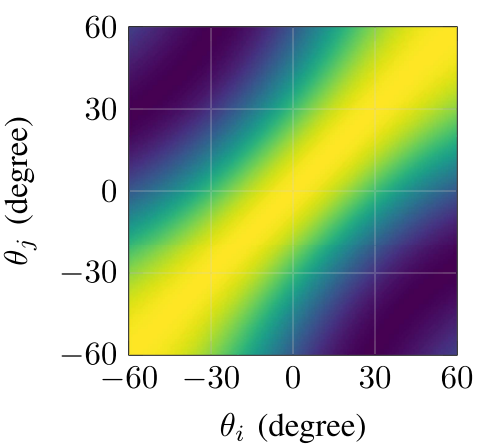

(a)

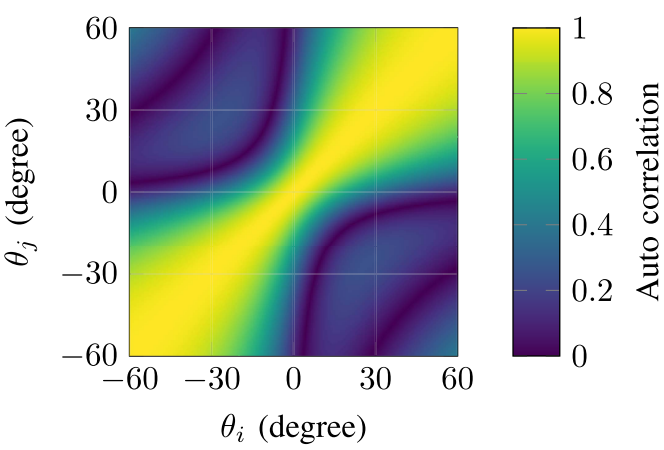

(b)

Fig. 4. Absolute value of the AF of (a) conventional, redundant virtual array and (b) biomimetic, nonredundant virtual array according to Fig. 3 with physical antenna element spacing of $d=0.37 \lambda$.

large incidence angles $\left(\theta \rightarrow 90^{\circ}\right)$, both patterns are similar because the phase gain reduces to around 1 .

\section{RADAR MMIC SYSTEM DESIGN}

The objective of this work was to show that biomimetic MIMO not only prevents redundant elements in the virtual aperture but also leads to very compact radar systems. An MMIC was developed in order to verify the principle of biomimetic MIMO. The concepts applied in the radar system design are described in this section.

\section{A. Radar System Concept}

As the antennas are usually the largest single component on an MMIC, it is desired to reduce their number to a minimum. Consequently, the presented MMIC is designed as a monostatic radar so that only one antenna is required for each of the two channels. The necessary transmit-receive coupler (TRC) is realized as an extremely compact rat-race coupler with one port terminated by $50 \Omega$ (see [11]). With its outer dimensions of $140 \mu \mathrm{m} \times 110 \mu \mathrm{m}$, the TRC only occupies approximately $10 \%$ of the area of an additional antenna, justifying the choice of the monostatic setup in order to be as compact as possible.

A simplified block diagram of the realized MMIC is shown in Fig. 5. The building blocks of the MMIC presented in this work are in parts based on the works in [11] and [26]. The MMIC incorporates an on-chip push-push-type voltagecontrolled oscillator (VCO) operating in the frequency range around $59 \mathrm{GHz}$ and is stabilized by an on-chip integer- $N$ phase-locked loop (PLL) (see the block "fixed-frequency PLL" in Fig. 5). As a reference frequency for the PLL, a signal in the frequency range of $920 \mathrm{MHz}$ has to be fed to the MMIC. The signal of the on-chip VCO is doubled in frequency and used to up-mix an FMCW ramp signal. This FMCW ramp signal, which has to be generated off-chip, has a center frequency of around $9 \mathrm{GHz}$ and its frequency is multiplied by a factor of four on-chip before upmixing. The signal generation forms an offset synthesizer with a low multiplication factor and is therefore optimized for low phase noise [4].

The synthesized signal in the $150-\mathrm{GHz}$ range is then divided into the two channels by an active power splitter (PS). In each channel, the signal is divided once again by the same type of PS into the transmit signal and the local oscillator (LO) signal.

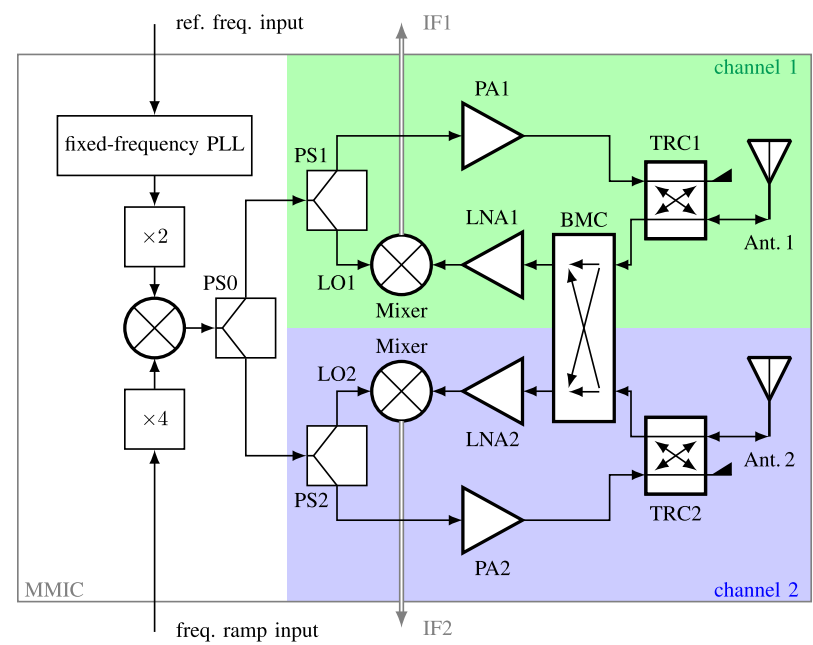

Fig. 5. Block diagram of the presented MMIC. Components belonging to channel 1 are highlighted in green, whereas channel 2 is marked in blue.

In the transmit path, a power amplifier (PA) is implemented, and afterward, the signal is directed to the TRC, where the signal is guided toward the on-chip antenna.

The signal received at each antenna is fed to the BMC network through the TRC. Thereafter, the received signal is amplified by a low-noise amplifier (LNA) and subsequently mixed down with the LO signal to the baseband [intermediate frequency (IF)].

The realized MMIC is shown in Fig. 6. It occupies a chip area of less than $3 \mathrm{~mm}^{2}$ with a length of $2.46 \mathrm{~mm}$ and a width of $1.21 \mathrm{~mm}$ and is realized in an $130-\mathrm{nm}$ SiGe-BiCMOS technology with an $f_{T} / f_{\max }$ of $300 / 500 \mathrm{GHz}$. Special emphasis was placed on routing the two channels of the MMIC symmetrically. In addition, the antennas and the bond connections were placed on different sides of the MMIC so that the mutual interference between them is minimized. The tight design of the MMIC allows for cascading, e.g., to build up larger radar systems.

\section{B. Integrated On-Chip Antennas and Biomimetic Coupling}

The integrated antennas of the MMIC are designed according to the work in [4], [27], and [28] and are composed of two parts. The first part is a shorted $\lambda / 4$-patch with a dimension 


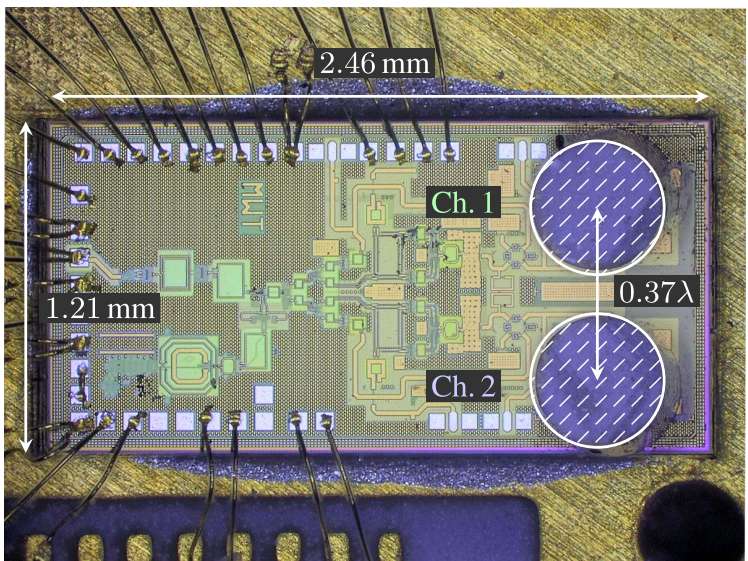

Fig. 6. Chip photograph of the bonded two-channel radar sensor with its outer dimensions. The chip is equipped with two DRAs placed on top of the on-chip patch antennas.

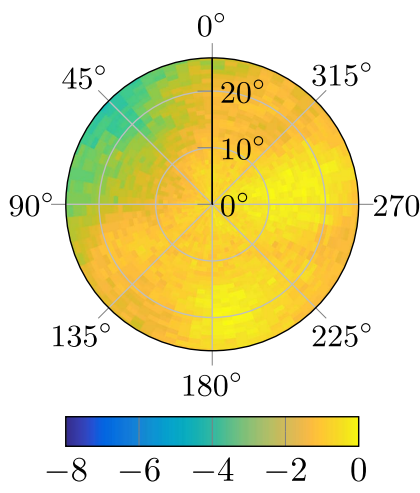

Norm. directivity $(\mathrm{dB})$

(a)

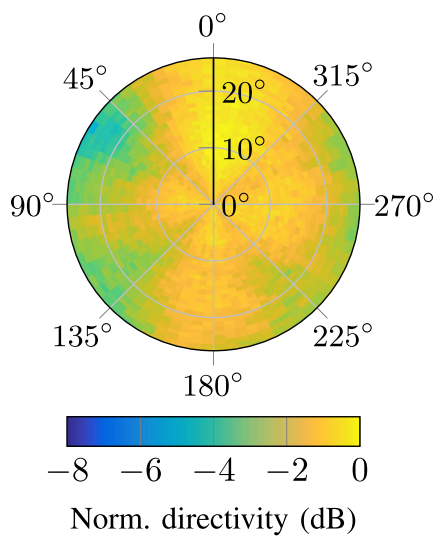

(b)
Fig. 7. Measured 3-D radiation patterns of the on-chip antennas of (a) channel 1 and (b) channel 2 at $150 \mathrm{GHz}$. The scanning plane of the radar ( $E$-plane) is oriented vertically and the $H$-plane is oriented horizontally.

of $220 \mu \mathrm{m} \times 180 \mu \mathrm{m}$, realized on the topmost layer of the back end of line (BEOL) of the semiconductor process. Two of these patches are spaced at a distance of $d=470 \mu \mathrm{m} \approx \lambda / 4$. The second component is a dielectric resonator antenna (DRA) with a diameter of $515 \mu \mathrm{m}$ and a height of $700 \mu \mathrm{m}$ placed on top of each of the structures to couple out the strong electric fields generated at the open end of the patch resulting in a spacing of $0.37 \lambda$ (see Fig. 6). The measured 3-D radiation patterns of both antennas at $150 \mathrm{GHz}$ are given in Fig. 7. The simulated maximum gain of each antenna is $3.8 \mathrm{dBi}$ at a $3-\mathrm{dB}$ beamwidth of $90^{\circ}$.

By using the method presented in Section II-A, a BMAA was built. The target design parameter were $\eta=3$ and $L_{\text {out }}=-9.5 \mathrm{~dB}$. Thus, the aperture is enlarged in the boresight direction by a factor of 3 , resulting in a total aperture size of $d_{\text {bio }} \approx \lambda$. The BMC network is designed according to the generalized model presented in [18] and is implemented in the RX path of the MMIC. A sketch of the layout of the realized BMC network is shown in Fig. 8. It consists of two vertically stacked microstrip line transformers on the two topmost metal layers of the semiconductor process. The antenna Y-parameters

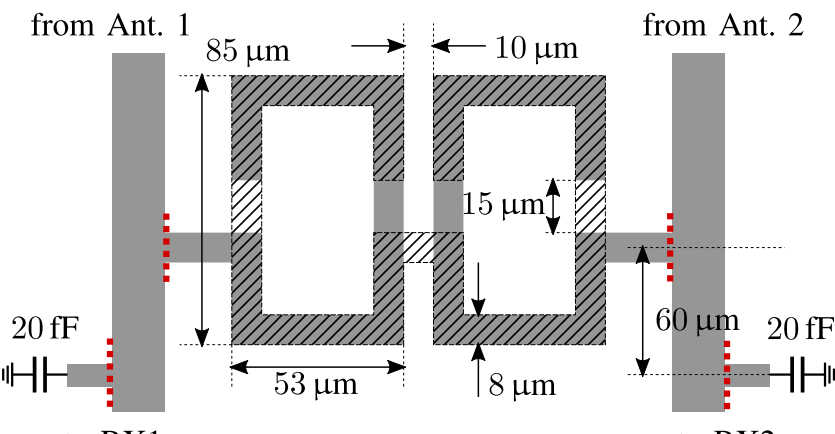

to $\mathrm{RX} 1$

Fig. 8. Layout of the BMC network consisting of two transformers realized on the MMIC. Structures on the topmost layer are displayed in gray, and structures located one layer below are shown in hatched. The BMC can be disconnected by cutting at the red dotted lines.

as the starting point of the design process were extracted from full-wave simulations. The required transmission characteristics of the BMC network, calculated by the design process, are then realized by adjusting the transmission factors from Ant. 1 to RX1 and from Ant. 1 to RX2. In order to realize the desired phase gain of $\eta=3$ for the presented antennas, the difference $\Delta$ in magnitude and phase of these transmission factors needs to fulfill $|\Delta|=-2.4 \mathrm{~dB}$ and $\arg (\Delta)=178.9^{\circ}$. The complete BMC network was optimized by full-wave simulations, and the two transformers were additionally measured on a separate breakout chip in order to confirm the simulations. A matching network using a shunt MIM capacitor is realized and placed at a distance of $60 \mu \mathrm{m}$ from the transformers toward the receivers. The coupling network is designed in a way that it can be disconnected by cutting the transformer feed lines as well as the matching stubs, e.g., with the focused ion beam (FIB) technology. This allows identifying the properties of MMICs equipped with BMAAs by comparing them to identical MMICs without biomimetic coupling.

\section{MIMO System Concept and Biomimetic MIMO}

Since the presented MMIC incorporates two transmitters and two receivers, a minimal MIMO system can be built up. The orthogonality of the TX signals is achieved by means of a time multiplexing by switching the PA in the TX path ON and OFF. A measured suppression of the TX signal of larger than $27 \mathrm{~dB}$ is achieved in the frequency band of interest by switching OFF the PA.

The redundant element in the virtual aperture-due to the monostatic configuration of the MMIC-is split into two separate elements by the biomimetic MIMO principle described in Section II-B. Thus, the overall aperture consists of four individual elements and is theoretically capable of separating three targets in the angular domain. Due to its increased phase progression, the size of the BMAA aperture also appears to be enlarged depending on the value of the phase gain [20] compared with the conventional array. In the boresight direction, the presented MIMO system, therefore, exhibits a total array size of $D=(\eta+1) d$, where $d=0.37 \lambda$ is the physical element separation on the realized MMIC. Due to a phase gain of $\eta=3$, the resulting MIMO aperture in 


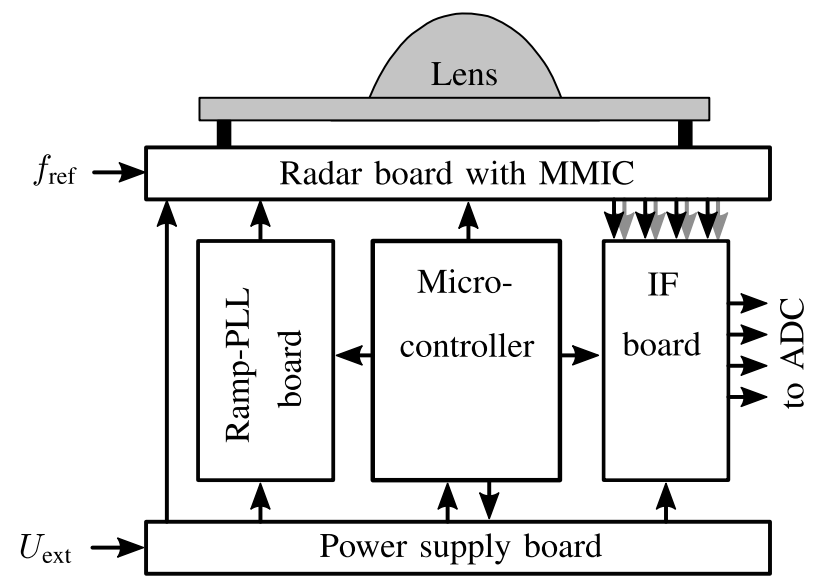

Fig. 9. Block diagram of the modular radar system. The radar MMIC is mounted on the radar board.

TABLE I

RADAR MODUlation PARAMETERS

\begin{tabular}{|l||c|}
\hline Ramp duration $T_{\mathrm{up}}$ & $100 \mu \mathrm{s}$ \\
\hline Ramp repetition time $T_{r}$ & $150 \mu \mathrm{s}$ \\
\hline Center frequency $f_{c}$ & $152.92 \mathrm{GHz}$ \\
\hline RF bandwidth $B$ & $8.24 \mathrm{GHz}$ \\
\hline Number of ramps $N_{r}$ & 512 \\
\hline
\end{tabular}

this work is doubled in size compared with the conventional MIMO aperture.

The theoretical diffraction limit for an antenna array to distinguish between two targets is given by the Rayleigh criterion [29]

$$
\Delta \theta=\frac{\lambda}{D}=\frac{\lambda}{(\eta+1) d}
$$

where $D$ is the total aperture size. With a designed phase gain of $\eta=3$ for the presented MMIC, a theoretical angular resolution of $\Delta \theta=38.7^{\circ}$ is possible compared with $\Delta \theta=154.8^{\circ}$ and $\Delta \theta=77.4^{\circ}$ for the conventional SIMO and MIMO array, respectively.

The application of BMAAs in a radar system is subject to a fundamental tradeoff, as already stated in Section II-A. Any increase in angle estimation performance will reduce the SNR by $L_{\text {out }}$, which in turn leads to a reduced range of the radar system. In general, due to the $R^{-4}$ dependence of the radar equation, the following relation can be used to estimate the reduction in maximum range $R_{\max }$ of the radar system assuming a required minimum receive power at the receiver

$$
R_{\text {max }, \mathrm{BMAA}}=\sqrt[4]{L_{\mathrm{out}}} R_{\text {max,conv }} .
$$

In the case under consideration, the reduction of the maximum range $R_{\max }$ for a designed $L_{\text {out }}=-9.5 \mathrm{~dB}$ is about $42 \%$ when applying BMAAs instead of a conventional array.

\section{Radar MeAsurements}

\section{A. Measurement Setup}

The developed MMIC was used in a modular radar system consisting of five stacked $\mathrm{PCBs}$, each providing a specific purpose. A block diagram of the system is given in Fig. 9. The radar MMIC presented in Section III is mounted on the

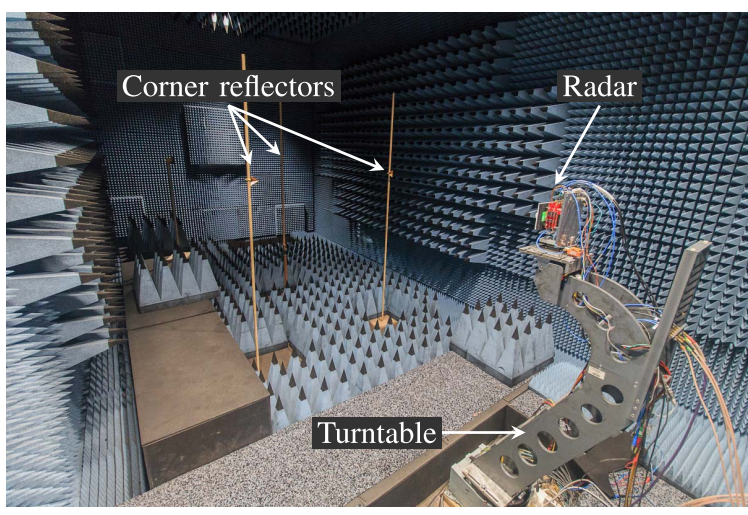

Fig. 10. Photograph of the measurement setup in an anechoic chamber used to characterize the radar system.

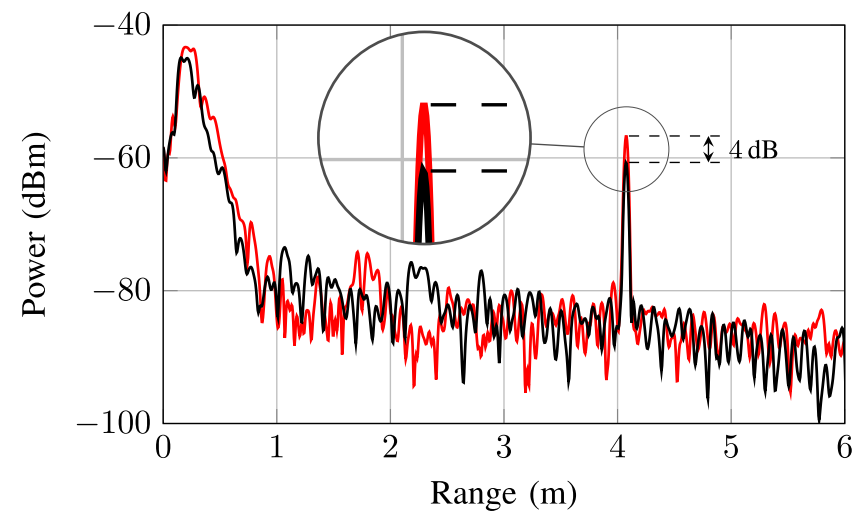

Fig. 11. Measured power range profile for one target at $\theta=0^{\circ}$ using the conventional (__ ) and the biomimetic (__ radar board. The signals of all four virtual channels were integrated noncoherently.

radar board, and a cylindrical lens is placed over the board to focus on the antenna beam in the elevation plane while leaving the beam in the azimuth plane unaltered. The resulting field of view (FOV) in the azimuth plane is $\pm 35^{\circ}$. The FMCW frequency ramp is generated with an off-the-shelf PLL with external VCO on the ramp-PLL board. The signals received by the radar are directed to the IF board, where the signals are filtered and amplified by a factor of up to $60 \mathrm{~dB}$ and subsequently forwarded to an external analog-to-digital converter (ADC). A microcontroller is used to program the modulation settings, modify the IF signal conditioning parameters, and control the radar MMIC. Two radar boards were built up in this work, one of which is carrying the unmodified MMIC (subsequently called biomimetic radar board), whereas the other one carries an MMIC with a disconnected BMC network (subsequently called conventional radar board).

The radar modulation parameters used in the measurements are given in Table I. All radar measurements were conducted in an anechoic chamber, while the radar stack was mounted on a turntable (see Fig. 10). Corner reflectors with different radar cross sections (RCSs) placed in the far field of the radar served as targets.

\section{B. Radar Measurements}

The system performance and the angular estimation performance are evaluated by radar measurements using a single 


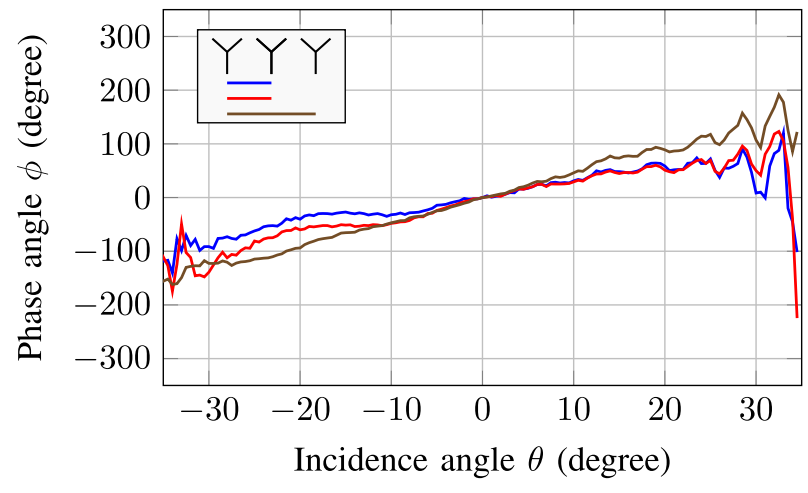

(a) Phase progression of conventional, redundant virtual array.

Auto correlation
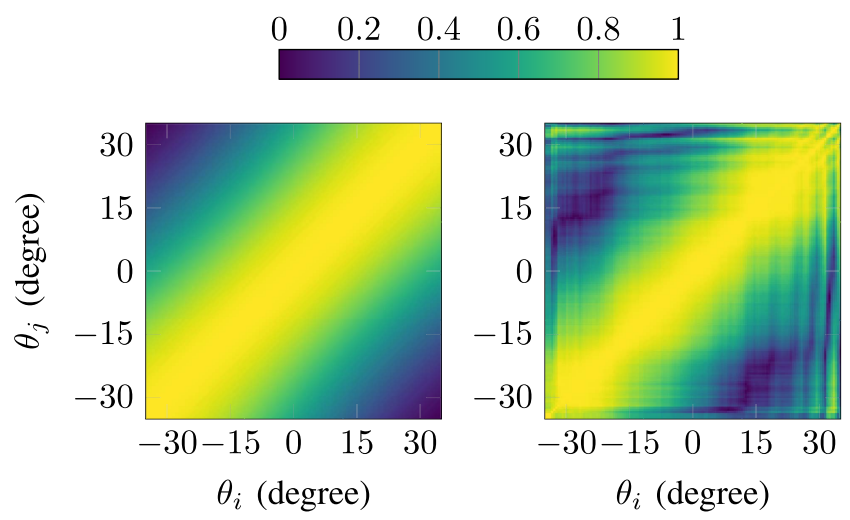

(c) Sim. (left) and meas. (right) AF of conventional, redundant virtual array.

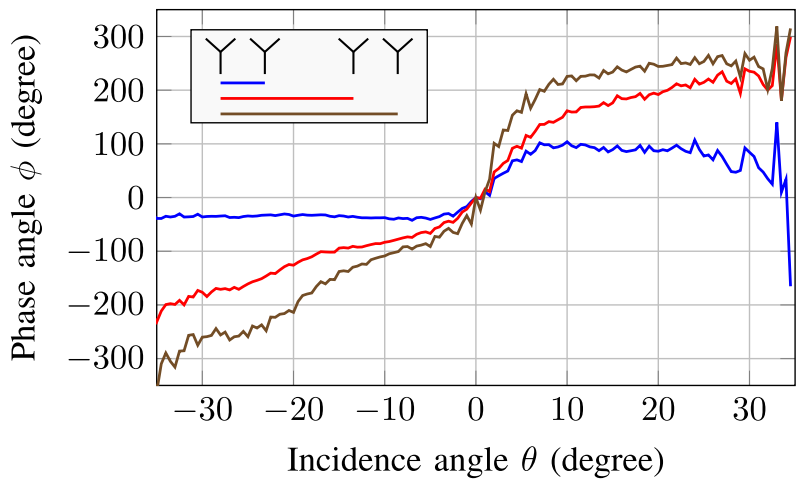

(b) Phase progression of biomimetic, non-redundant virtual array with $\eta=3$

Auto correlation

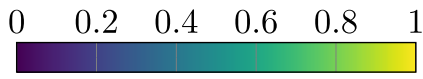

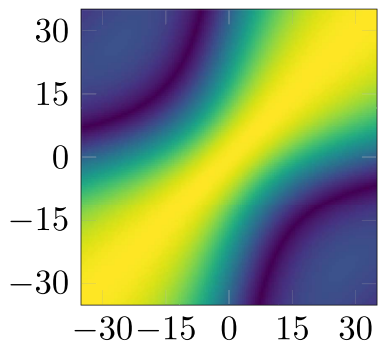

$\theta_{i}$ (degree)

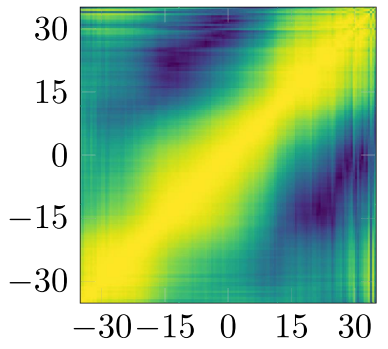

$\theta_{i}$ (degree) (d) Sim. (left) and meas. (right) AF of biomimetic, non-redundant virtual array.

Fig. 12. Measured phase progressions normalized to the first array element for (a) conventional and (b) biomimetic aperture and simulated and measured absolute value of the AF for (c) conventional and (d) biomimetic aperture.

TABLE II

Normalized OUtPut PoWER LEVEL $L_{\text {OUt }}$ OF EVERy VIRTUAL CHANNEL FOR A TARGET AT $\theta=0^{\circ}$

\begin{tabular}{|c||c|c|c|c|}
\hline & chan. 1 & chan. 2 & chan. 3 & chan. 4 \\
\hline$L_{\text {out }}$ & $-0.4 \mathrm{~dB}$ & $-6.4 \mathrm{~dB}$ & $-12.3 \mathrm{~dB}$ & $-5.9 \mathrm{~dB}$ \\
\hline
\end{tabular}

target at a distance of around $4 \mathrm{~m}$ at boresight $\left(\theta=0^{\circ}\right)$. Fig. 11 shows the power range profile of both radar boards under test. The IF signals of all four virtual channels are integrated noncoherently. It can be noticed that the overall shape of the graphs and the noise floor is comparable among the two boards, whereas the biomimetic radar board shows a 4-dB lower target power level. However, the reduction of the power level is lower than the designed $L_{\text {out }}$ value of $-9.5 \mathrm{~dB}$. Table II lists the $L_{\text {out }}$ values for each individual virtual channel determined by the target peak difference of the measurements with the biomimetic and the conventional radar board. While, in theory, the normalized output power is the same for every virtual channel, deviations can be found in the measurements. This is due to fabrication tolerances, e.g., the placement of the DRAs on the MMICs and parasitic influences of the measurement setup. By integrating the values of Table II, the measured reduction of $4 \mathrm{~dB}$ is obtained. Referring to the weakest of the measured virtual channels, the maximum range $R_{\max }$ of the biomimetic radar board according to (13) is reduced by $50 \%$ compared with the conventional radar board.
In a next step, the radar was rotated around its axis with the target fixed at $0^{\circ}$. A radar measurement was performed for every angle in the range $\theta=-35^{\circ} \ldots 35^{\circ}$ with a step size of $0.5^{\circ}$. For each measurement and for every virtual channel, the phase at the target location was extracted from the power range profile, and the phase progression between the respective virtual channels was calculated. Fig. 12(a) and (b) shows the measured phase progressions normalized to the first virtual array element using the conventional and the biomimetic radar board, respectively. The effect of the biomimetic coupling can clearly be noticed as the single curves in Fig. 12(b) show a significantly steeper slope in the vicinity of boresight. It can also be noticed that Fig. 12(a) shows three curves, but two of them lie almost on top of each other. This means that a redundant element is present for the conventional radar board, as predicted in Section II. In Fig. 12(b), this redundancy is no more visible as all three curves show a unique course and are clearly separable. Thus, the redundancy of the monostatic MIMO radar has been eliminated by using the biomimetic radar board.

From the measured phase progressions, the ambiguity diagrams for both radar systems under test were calculated according to (11) and are shown in Fig. 12(c) and (d). While the conventional radar board shows a constant width of the main beam, a clear constriction can be noticed for the biomimetic radar board, especially for $\theta>0^{\circ}$. This originates from the steeper phase progression and is leading to a smaller 


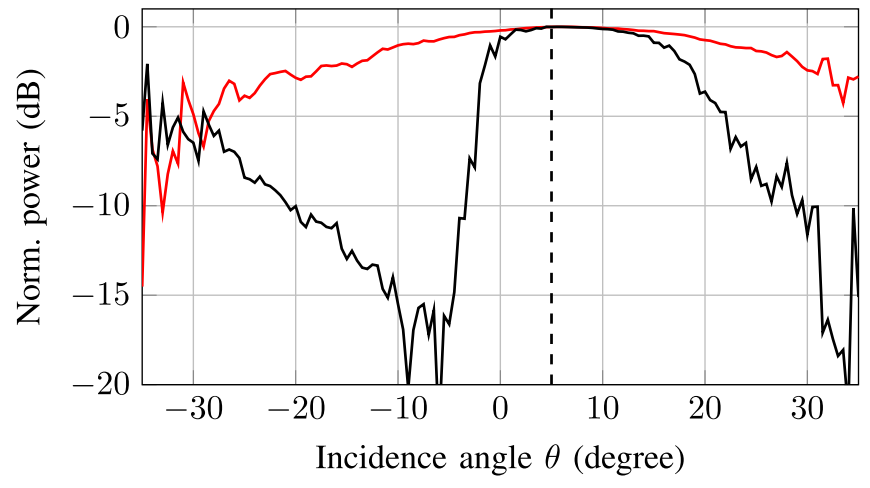

Fig. 13. Measured angular estimation performance of the conventional (__ ) and the biomimetic (__ ) MIMO radar system for one target at $\theta=5^{\circ}$. The true DOA is marked with a dashed line.

lobe in the angle estimation. The experimental results are in good agreement with the theoretical findings in Fig. 4.

For a target placed at an angle of $5^{\circ}$ at a distance of $3 \mathrm{~m}$ to the radar, the angle estimation spectra are shown in Fig. 13 for the conventional and the biomimetic radar. A maximum likelihood (ML) method was used for the angle estimation in which the measured data are correlated with a prerecorded calibration vector of all incidence angles $\theta$ and normalized to $0 \mathrm{~dB}$. The significantly narrower lobe of the ambiguity plot can also be noticed here, leading to a more accurate angle estimation. A statistical study on the angle estimation accuracy by performing 100 measurements with only one transmitter being active (SIMO case) was already presented in [26] showing an improvement in angular accuracy of around a factor of 2 for a phase gain of $\eta=4$.

\section{CONCLUSION}

This contribution proposed biomimetic MIMO as a fundamentally new MIMO principle based on the concept of biomimetic antenna arrays to overcome the problem of redundant elements in monostatic MIMO systems. The new MIMO principle was applied to an ultracompact monostatic radar based on an MMIC in the $150-\mathrm{GHz}$ range with two transmit and receive channels occupying a chip area of $3 \mathrm{~mm}^{2}$ only. Radar measurements utilizing the presented MMIC verified the working principle of the new MIMO concept. A virtual aperture consisting of four unique antenna positions and an equivalent total size of around $1.5 \lambda$ was obtained. The presented concept is not limited to monostatic setups but also holds for bistatic MIMO radars where the TX and RX antennas are spaced identically.

\section{APPENDIX}

\section{DERIVATION OF FORMULA FOR $L_{\text {OUT }}$}

The normalized output power $L_{\text {out }}$ of the BMAA is defined as [18]

$$
L_{\text {out }}=\left|\frac{u_{i, \text { BMAA }}}{u_{i, \text { conv. }}}\right|^{2} \text { with } i=1,2
$$

where $u_{i \text {,BMAA }}$ and $u_{i \text {,conv. }}$ are the voltages at the antenna system output for the biomimetic and the conventional antenna array, respectively, and $i$ indicates the corresponding antenna port. The two voltages have been derived in [18] as

$$
\begin{aligned}
u_{i, \text { BMAA }} & =\frac{ \pm \mathrm{j} A_{0} \sin \alpha}{Y_{11}-Y_{12}+G_{L}}+\frac{A_{0} \cos \alpha}{Y_{11}+Y_{12}+G_{L}+G_{C}+\mathrm{j} B_{C}} \\
u_{i, \text { conv. }} & =\frac{ \pm \mathrm{j} A_{0} \sin \alpha}{Y_{11}-Y_{12}+G_{L}}+\frac{A_{0} \cos \alpha}{Y_{11}+Y_{12}+G_{L}}
\end{aligned}
$$

depending on the antenna Y-parameters ( $Y_{11}$ and $\left.Y_{12}\right)$, the load $\left(G_{L}\right)$, and the lumped elements of the coupling network $\left(G_{C}\right.$ and $\left.B_{C}\right)$. The coupling network elements $G_{C}$ and $B_{C}$ are not present for the conventional antenna array, i.e., $G_{C}=B_{C}=0$. Inserting into (14), rearranging, and introducing the variable $z$ as

$$
z=\eta+\mathrm{j} \xi=\frac{Y_{11}+Y_{12}+G_{L}+G_{C}+\mathrm{j} B_{C}}{Y_{11}-Y_{12}+G_{L}}
$$

the following equation for $L_{\text {out }}$ is derived:

$$
L_{\text {out }}=\left|\frac{\frac{1}{z} \pm \mathrm{j} \tan \alpha}{1+\mathrm{j} \tan \alpha}\right|^{2}=\left|\frac{1 \pm \mathrm{j}(\eta+\mathrm{j} \xi) \tan \alpha}{\eta+\mathrm{j}(\xi+(\eta+\mathrm{j} \xi) \tan \alpha)}\right|^{2} .
$$

\section{REFERENCES}

[1] M. Schröter et al., "SiGe HBT technology: Future trends and TCADbased roadmap," Proc. IEEE, vol. 105, no. 6, pp. 1068-1086, Jun. 2017.

[2] S. P. Voinigescu, S. Shopov, J. Bateman, H. Farooq, J. Hoffman, and K. Vasilakopoulos, "Silicon millimeter-wave, terahertz, and high-speed fiber-optic device and benchmark circuit scaling through the 2030 ITRS horizon," Proc. IEEE, vol. 105, no. 6, pp. 1087-1104, Jun. 2017.

[3] I. Sarkas, J. Hasch, A. Balteanu, and S. P. Voinigescu, "A fundamental frequency $120-\mathrm{GHz}$ SiGe BiCMOS distance sensor with integrated antenna," IEEE Trans. Microw. Theory Techn., vol. 60, no. 3, pp. 795-812, Mar. 2012.

[4] M. Hitzler et al., "Ultracompact 160-GHz FMCW radar MMIC with fully integrated offset synthesizer," IEEE Trans. Microw. Theory Techn., vol. 65, no. 5, pp. 1682-1691, May 2017.

[5] A. Mostajeran, A. Cathelin, and E. Afshari, "A 170-GHz fully integrated single-chip FMCW imaging radar with 3-D imaging capability," IEEE J. Solid-State Circuits, vol. 52, no. 10, pp. 2721-2734, Oct. 2017.

[6] S. Thomas, C. Bredendiek, T. Jaeschke, F. Vogelsang, and N. Pohl, "A compact, energy-efficient $240 \mathrm{GHz}$ FMCW radar sensor with high modulation bandwidth," in Proc. German Microw. Conf. (GeMiC), Bochum, Germany, Mar. 2016, pp. 397-400.

[7] H. J. Ng, M. Kucharski, W. Ahmad, and D. Kissinger, "Multi-purpose fully differential $61-$ and $122-\mathrm{GHz}$ radar transceivers for scalable MIMO sensor platforms," IEEE J. Solid-State Circuits, vol. 52, no. 9, pp. 2242-2255, Sep. 2017.

[8] A. Mostajeran et al., "A high-resolution 220-GHz ultra-wideband fully integrated ISAR imaging system," IEEE Trans. Microw. Theory Techn., vol. 67, no. 1, pp. 429-442, Jan. 2019.

[9] M. G. Girma, J. Hasch, I. Sarkas, S. P. Voinigescu, and T. Zwick, "122 GHz radar sensor based on a monostatic SiGe-BiCMOS IC with an on-chip antenna," in Proc. 7th Eur. Microw. Integr. Circuit Conf., Amsterdam, The Netherlands, Oct. 2012, pp. 357-360.

[10] E. Özturk et al., "A $120 \mathrm{GHz}$ SiGe BiCMOS monostatic transceiver for radar applications," in Proc. 13th Eur. Microw. Integr. Circuits Conf. (EuMIC), Madrid, Spain, Sep. 2018, pp. 41-44.

[11] M. Hitzler, P. Grüner, L. Boehm, W. Mayer, and C. Waldschmidt, "On monostatic and bistatic system concepts for mm-wave radar MMICs," IEEE Trans. Microw. Theory Techn., vol. 66, no. 9, pp. 4204-4215, Sep. 2018.

[12] S. Shopov, M. G. Girma, J. Hasch, N. Cahoon, and S. P. Voinigescu, "Ultralow-power radar sensors for ambient sensing in the $V$-Band," IEEE Trans. Microw. Theory Techn., vol. 65, no. 12, pp. 5401-5410, Dec. 2017.

[13] E. Öztürk et al., "A $60 \mathrm{GHz}$ SiGe BiCMOS monostatic transceiver for radar applications," in IEEE MTT-S Int. Microw. Symp. Dig., Honololu, HI, USA, Jun. 2017, pp. 1408-1411. 
[14] M. G. Girma, M. Gonser, A. Frischen, J. Hasch, Y. Sun, and T. Zwick, "122 GHz single-chip dual-channel SMD radar sensor with on-chip antennas for distance and angle measurements," Int. J. Microw. and Wireless Techn., vol. 7, nos. 3-4, pp. 407-414, Jun. 2015.

[15] H. J. Ng and D. Kissinger, "Highly miniaturized 120-GHz SIMO and MIMO radar sensor with on-chip folded dipole antennas for range and angular measurements," IEEE Trans. Microw. Theory Techn., vol. 66, no. 6, pp. 2592-2603, Jun. 2018.

[16] R. N. Miles, D. Robert, and R. R. Hoy, "Mechanically coupled ears for directional hearing in the parasitoid fly ormia ochracea," J. Acoust. Soc. Amer., vol. 98, no. 6, pp. 3059-3070, Dec. 1995.

[17] A. R. Masoumi, Y. Yusuf, and N. Behdad, "Biomimetic antenna arrays based on the directional hearing mechanism of the parasitoid fly ormia ochracea," IEEE Trans. Antennas Propag., vol. 61, no. 5, pp. 2500-2510, May 2013.

[18] P. Grüner, T. Chaloun, and C. Waldschmidt, "A generalized model for two-element biomimetic antenna arrays," IEEE Trans. Antennas Propag., vol. 67, no. 3, pp. 1630-1639, Mar. 2019.

[19] M. Ranjbar Nikkhah, K. Ghaemi, and N. Behdad, "An electronically tunable biomimetic antenna array," IEEE Trans. Antennas Propag., vol. 66, no. 3, pp. 1248-1257, Mar. 2018.

[20] P. Grüner, T. Chaloun, and C. Waldschmidt, "Enhanced angle estimation accuracy of ultra compact radars inspired by a biomimetic approach," in IEEE MTT-S Int. Microw. Symp. Dig., Honolulu, HI, USA. Jun. 2017, pp. $1425-1428$.

[21] J. Li and P. Stoica, MIMO Radar Signal Processing. Hoboken, NJ, USA: Wiley, 2009.

[22] A. R. Masoumi and N. Behdad, "An improved architecture for twoelement biomimetic antenna arrays," IEEE Trans. Antennas Propag., vol. 61, no. 12, pp. 6224-6228, Dec. 2013.

[23] P. Grüner, S. Nguyen, T. Chaloun, and C. Waldschmidt, "Enhancing angle estimation for off-boresight targets using biomimetic antenna arrays," in Proc. 48th Eur. Microw. Conf. (EuMC), Madrid, Spain, Sep. 2018, pp. 1377-1380.

[24] F. C. Robey, S. Coutts, D. Weikle, J. C. McHarg, and K. Cuomo, "MIMO radar theory and experimental results," in Proc. 28th Asilomar Conf. Signals, Syst. Comput., Pacific Grove, CA, USA. 2004, pp. 300-304.

[25] M. Eric, A. Zejak, and M. Obradovic, "Ambiguity characterization of arbitrary antenna array: Type I ambiguity," in Proc. IEEE 5th Int. Symp. Spread Spectr. Techn. Appl., Sun City, South Africa, vol. 2, Sep. 1998, pp. 399-403.

[26] P. Grüner, T. Chaloun, and C. Waldschmidt, "Enhancing angle estimation accuracy of ultra compact two-channel radar MMICs at $160 \mathrm{GHz}$ using a biomimetic antenna array," in IEEE MTT-S Int. Microw. Symp. Dig., Boston, MA, USA, Jun. 2019, pp. 305-308.

[27] M. Hitzler and C. Waldschmidt, "Design and characterization concepts of a broadband chip-integrated antenna," in Proc. 44th Eur. Microw. Conf., Rome, Italy, Oct. 2014, pp. 96-99.

[28] J. Hasch, U. Wostradowski, S. Gaier, and T. Hansen, "77 GHz radar transceiver with dual integrated antenna elements," in Proc. German Microw. Conf. (GeMiC), Berlin, Germany, Mar. 2010, pp. 280-283.

[29] H. L. Van Trees, Optimum Array Processing (Detection, Estimation, and Modulation Theory), vol. 4. Hoboken, NJ, USA: Wiley, 2002.

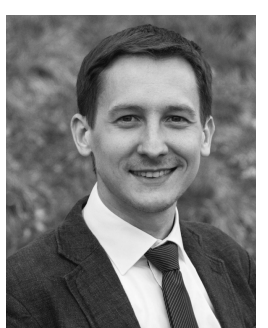

Patrik Grüner (Graduate Student Member, IEEE) received the M.Sc. degree in electrical engineering from Ulm University, Ulm, Germany, in 2015, where he is currently pursuing the Ph.D. degree.

In 2015, he joined the Institute of Microwave Engineering (MWT), Ulm University. His current research interests include compact frequencymodulated continuous-wave radar sensors and biomimetic antenna systems both in the microwave and millimeter-wave ranges

Mr. Grüner was a recipient of the ARGUS Science Award in 2013 and the Best Paper Award of the 2013 International Workshop on Antenna Technology.

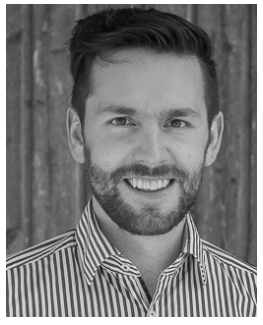

Martin Geiger (Graduate Student Member, IEEE) received the M.Sc. degree from Ulm University, Ulm, Germany, in 2015, where he is currently pursuing the $\mathrm{Ph} . \mathrm{D}$. degree.

In 2016, he joined the Institute of Microwave Engineering, Ulm University. His current research interests include novel radar sensor concepts with flexible antennas, dielectric waveguides, and monolithic microwave integrated circuit (MMIC) interconnects, all at millimeter-wave frequencies.

Mr. Geiger was a recipient of the Best Student Paper Award of the 2018 International Microwave Symposium.

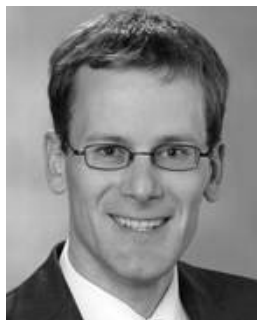

Christian Waldschmidt (Senior Member, IEEE) received the Dipl.Ing. (M.S.E.E.) and Dr.Ing. (Ph.D.E.E.) degrees from the University of Karlsruhe (TH), Karlsruhe, Germany, in 2001 and 2004, respectively.

From 2001 to 2004, he was a Research Assistant with the Institut für Höchstfrequenztechnik and Elektronik (IHE), TH. Since 2004, he has been with Robert Bosch GmbH, Stuttgart, Germany, in the business units Corporate Research and Chassis Systems. He was heading different research and development teams in microwave engineering, RF sensing, and automotive radar. In 2013, he returned to academia. He was appointed as the Director of the Institute of Microwave Engineering, University Ulm, Ulm, Germany, where he is currently a Full Professor. He authored or coauthored over 200 scientific publications and more than 20 patents. His research interests include radar and RF sensing, millimeter-wave and submillimeter-wave engineering, antennas and antenna arrays, RF, and array signal processing.

Dr. Waldschmidt is a member of the Executive Committee Board of the German MTT/AP Joint Chapter and the German Information Technology Society (ITG). He was a co-recipient of 11 best paper awards since 2014 . He has served as the Chair for the IEEE MTT-27 Technical Committee on Wireless Enabled Automotive and Vehicular Applications. He was a two-time TPC Chair and the General Chair of the IEEE MTT International Conference on Microwaves for Intelligent Mobility. He is also a reviewer for multiple IEEE TRANSACTIONS and many IEEE conferences in the field of microwaves. Since 2018, he has been serving as an Associate Editor for the IEEE MTT MicrowaVe Wireless COMPONENTS LETTERS (MWCL). 